University of Nebraska - Lincoln

DigitalCommons@University of Nebraska - Lincoln

Fall 9-4-2008

\title{
Electrically Controlled Magnetism
}

\author{
Christian Binek \\ University of Nebraska, Lincoln, cbinek@unl.edu \\ Xi He \\ University of Nebraska-Lincoln, lodgehe@bigred.unl.edu \\ Yi Wang \\ ywang1983@hotmail.com \\ Sarbeswar Sahoo \\ University of Nebraska-Lincoln, sarbeswar@gmail.com
}

Follow this and additional works at: https://digitalcommons.unl.edu/physicsbinek

Part of the Physics Commons

Binek, Christian; He, Xi; Wang, Yi; and Sahoo, Sarbeswar, "Electrically Controlled Magnetism" (2008). Christian Binek Publications. 65.

https://digitalcommons.unl.edu/physicsbinek/65

This Article is brought to you for free and open access by the Research Papers in Physics and Astronomy at DigitalCommons@University of Nebraska - Lincoln. It has been accepted for inclusion in Christian Binek Publications by an authorized administrator of DigitalCommons@University of Nebraska - Lincoln. 


\title{
Electrically Controlled Magnetism
}

\author{
Ch. Binek, Xi He, Yi Wang, and S. Sahoo \\ Department of Physics and Astronomy and the Nebraska Center for Materials and Nanoscience, \\ University of Nebraska, Lincoln, Nebraska 68588, USA
}

\begin{abstract}
Manipulation of magnetically ordered states by electrical means is a promising approach towards novel spintronics devices. We report on the electric control of surface magnetism in $\mathrm{Cr}_{2} \mathrm{O}_{3}$ thin films and uniaxial anisotropy in ferroelectric/ferromagnetic heterostructures, respectively. Artificial magnetoelectricity is realized in a $\mathrm{BaTiO}_{3} / \mathrm{Fe}$ heterostructure. Here, thermally induced coercivity changes of the Fe hysteresis loop are used to derive the stress imposed by the ferroelectric $\mathrm{BaTiO}_{3}$ substrate on the adjacent Fe film. Electrically induced coercivity changes give rise to a giant magnetoelectric susceptibility in the vicinity of the magnetic coercive field.
\end{abstract}

Keywords: magnetoelectrics, multiferroics, spintronics, and magnetic anisotropy

7036-32 V. 2 (p.1 of 9) / Color: No / Format: Letter / Date: 7/8/2008 3:57:50 PM 


\section{Introduction}

Electric control of magnetism is promising for technologically novel device paradigms. In this regard, tremendous attention is being focused on two kinds of materials. First, magnetoelectric materials ${ }^{1,2}$ where a linear magnetic response $M^{i}$ (electric response $P^{i}$ ) can be induced by the application of an electric field $E_{j}$ (magnetic field $H_{j}$ ) such that $\mu_{0} M^{i}=\alpha_{m e}^{i j} E_{j}$ and $P^{i}=\alpha_{e m}^{i j} H_{j}$, where $\alpha_{m e}^{i j}=\alpha_{e m}^{j i}$ are the tensors of ME susceptibility and its transpose counterpart while $i, j$ label the vector and tensor components, respectively. In particular coupling with an electric field can take place in the absence of spontaneous polarization. Second, multiferroics ${ }^{3}$ where both the electric and magnetic spontaneous orders coexist and can be manipulated by conjugate fields. Of particular interest is coupling of the ferroic order parameters such that these multiferroics also show a magnetoelectric effect. The magnetoelectric antiferromagnets and magnetoelectric multiferroics ${ }^{4}$ reveal exciting physical phenomena from the scientific perspectives and have the potential to revolutionize future spintronic devices. ${ }^{5}$ Recent resurgence of research and development ${ }^{6}$ activities on extrinsic control of the coupling between the primary ferroic (magnetic, electric, and elastic) order parameters is motivated by the possibility to design new devices that are compact, less power consuming and cheaper. Switching of a ferromagnetic (FM) by an electric field for instance promises significant impact in the design of next generation spintronic devices such as high density electric-field controlled MRAM. ${ }^{7}$

Most recently studied multiferroics and magnetoelectrics can be classified into two kinds of systems: singlephase systems and two-phase systems. Single-phase magnetoelectric multiferroics ${ }^{8,9,10,11,12,13}$ are predicted to be rare due to symmetry constraints. ${ }^{14}$ Compounds know today exhibit complex chemical and structural properties and show relatively small coupling, $\alpha=(\partial P / \partial H)_{E}=\mu_{0}(\partial M / \partial E)_{H} \approx 10^{-12} \mathrm{sm}^{-1}$. (Refs. 4,15,16) Two-phase systems ${ }^{17,18,19,20,21,22,23}$ include artificially grown ferroelectric (FE)-FM heterostructures such as $\mathrm{BaTiO}_{3} / \mathrm{La}_{0.66} \mathrm{Sr}_{0.33} \mathrm{MnO}_{3}(\mathrm{BTO} / \mathrm{LSMO}$ ), $\mathrm{BiFeO}_{3} / \mathrm{SrTiO}_{3}$, nano-pillar embedded structures of $\mathrm{BTO}-\mathrm{CoFe}_{2} \mathrm{O}_{4}$ or $\mathrm{BiFeO}_{3}-\mathrm{CoFe}_{2} \mathrm{O}_{4}, \mathrm{~Pb}(\mathrm{Zr}, \mathrm{Ti}) \mathrm{O}_{3} / \mathrm{CoPd}$, $\mathrm{Pb}(\mathrm{Zr}, \mathrm{Ti}) \mathrm{O}_{3} /$ terfenol-D etc. They show a relatively large coupling constant, $\alpha \approx 10^{-7} \mathrm{sm}^{-1}$ and can be promising candidates for applications. ${ }^{16}$

In this paper, we deal with two well-known classical magnetoelectric and multiferroic systems and show how these prototypical compounds and heterosystems enable electric control of magnetism. Bulk $\mathrm{Cr}_{2} \mathrm{O}_{3}$ is the prototypical ME antiferromagnet. Its Néel temperature of $T_{\mathrm{N}}=307 \mathrm{~K}$ makes $\mathrm{Cr}_{2} \mathrm{O}_{3}$ a promising candidate for room temperature applications. Indeed, prototypical $\mathrm{Cr}_{2} \mathrm{O}_{3}$ (111)/CoPt heterostructures ${ }^{24,25}$ based on (111) oriented $\mathrm{Cr}_{2} \mathrm{O}_{3}$ bulk single crystals have been successfully studied to control the magnetic exchange bias effect ${ }^{26}$ via electric fields. In order to fabricate spintronic devices as such proposed in Ref. 5, highest quality $\mathrm{Cr}_{2} \mathrm{O}_{3}$ (111) thin films have to be grown in proximity of metallic thin films. $\mathrm{Cr}_{2} \mathrm{O}_{3}$ in its thin film form can have markedly different magnetic as well as magnetoelectric properties from its bulk counterpart. Thus, systematic investigation of $\mathrm{Cr}_{2} \mathrm{O}_{3}$ thin film is an important step towards heterostructures functionalized for spintronic applications. Here we explicitly focus on the growth, surface magnetic and magnetoelectric properties of $\mathrm{Cr}_{2} \mathrm{O}_{3}$ films.

Our second system corresponds to a generic two-phase multiferroic system comprised of a transition metal FM Fe thin film on a single-crystal $\mathrm{BaTiO}_{3}$ substrate. We systematically explore the modification in Fe film magnetism as BTO undergoes various structural phase transitions as well as the so-called converse magnetoelectric effect in this novel heterostructure. Particular emphasis lies on the accompanying magnetoresistive transitions. The observed effects are significantly enhanced compared to other multiferroics involving complex FM oxides.

\section{Growth, structural, magnetic and magnetoelectric properties of $\mathrm{Cr}_{2} \mathrm{O}_{3}$ thin films}

$\mathrm{Cr}_{2} \mathrm{O}_{3}$ thin films are grown on top of a single crystalline (110) $\mathrm{Cr}$ seed layer by Molecular Beam Epitaxy at a base pressure of $2 \times 10^{-11}$ mbar. Since $\mathrm{Cr}$ exhibits multiple oxide states $(+3,+4,+6)$, previous studies reveal that stringent control of the oxygen partial pressure and substrate temperature during the evaporation of $\mathrm{Cr}$ metal is to be fulfilled in order to optimize the growth of stoichiometric $\mathrm{Cr}_{2} \mathrm{O}_{3}$ films. ${ }^{27}$ Metallic $\mathrm{Cr}$ is evaporated and transformed into the stable $\mathrm{Cr}_{2} \mathrm{O}_{3}$ oxide using an oxygen partial pressure of $2.2 \times 10^{-6}$ mbar during deposition while the substrate is maintained at $T=$ $573 \mathrm{~K}$. At this growth conditions, the growth rate of $\mathrm{Cr}_{2} \mathrm{O}_{3}$ films monitored by a calibrated quartz oscillator was found to be $0.28 \mathrm{~nm} / \mathrm{min}$. The resulting $\mathrm{Cr}_{2} \mathrm{O}_{3}$ film was capped with another $\mathrm{Cr}$ layer for the purpose of applying electric fields across the $\mathrm{Cr}_{2} \mathrm{O}_{3}$ film. The samples are structurally characterized by small and large angle X-ray diffraction (XRD). Figure 1 shows the large angle XRD pattern of $\mathrm{Cr}_{2} \mathrm{O}_{3}$ film grown on $c-\mathrm{Al}_{2} \mathrm{O}_{3} / \mathrm{Cr}(6 \mathrm{~nm})$. Analysis of these data by using rhombohedral unit cell parameters shows predominantly (111)-oriented epitaxial growth of the $\mathrm{Cr}_{2} \mathrm{O}_{3}$ film. The inset shows the small angle X-ray reflectometry (SAXR) curve of $\mathrm{Al}_{2} \mathrm{O}_{3} / \mathrm{Cr} / \mathrm{Cr}_{2} \mathrm{O}_{3} / \mathrm{Cr}$ structure. Two different kinds of 
periodic Bragg oscillations are clearly resolved. Small and large oscillations correspond to the total film thickness and top Cr layer thickness, respectively. Analysis of the SAXR fit yields the total $\mathrm{Cr} / \mathrm{Cr}_{2} \mathrm{O}_{3} / \mathrm{Cr}$ thickness as $117 \mathrm{~nm}$ and the top $\mathrm{Cr}$ film thickness as $6 \mathrm{~nm}$. The mean value of surface roughness was $\sim 0.4 \mathrm{~nm}$.

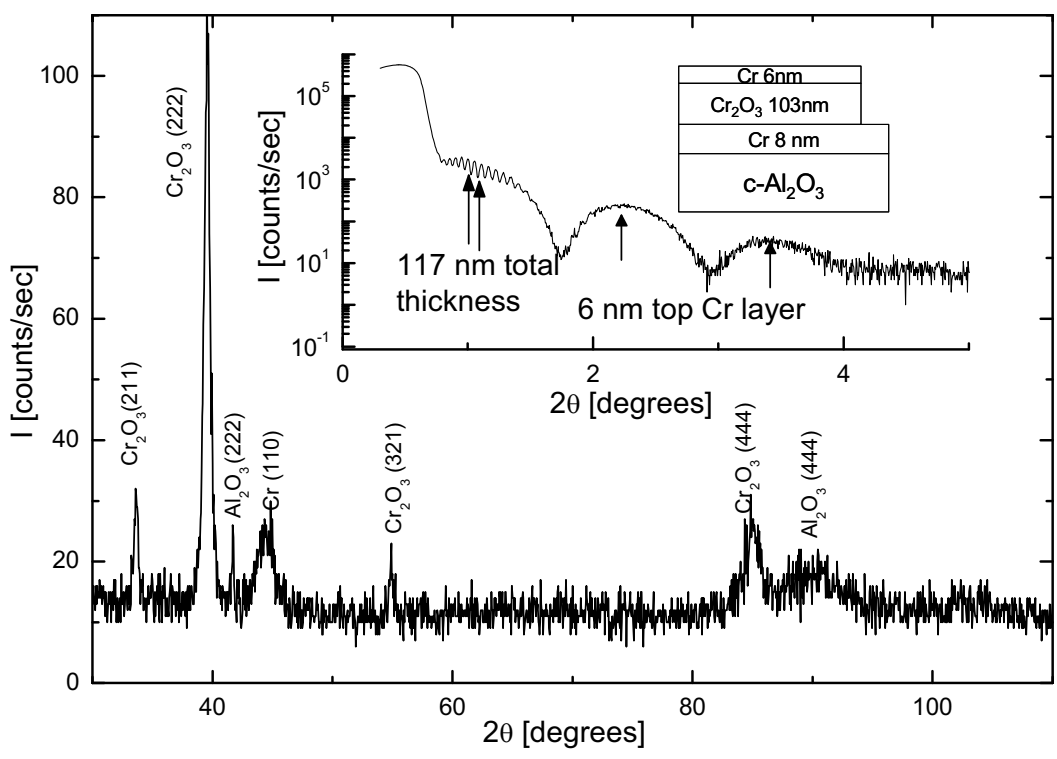

Fig. 1: X-ray diffraction pattern of $\mathrm{Cr} / \mathrm{Cr}_{2} \mathrm{O}_{3} / \mathrm{Cr}$ trilayer. The inset shows small angle X-ray reflectivity data. Small and large oscillations yield a measure of the total thickness and top Cr layer thickness, respectively. A sketch of the heterolayers is given as an inset.

Figure 2 shows the temperature dependence of the polar Kerr signal measured after various magnetoelectric annealing protocols applied to the $\mathrm{Cr} / \mathrm{Cr}_{2} \mathrm{O}_{3} / \mathrm{Cr}$ film. Squares show the remanent Kerr signal vs. temperature after field cooling the sample exclusively in an axial applied magnetic field of $0.05 \mathrm{~T}$. Circles and triangles represent the remanent Kerr signal after magnetoelectric annealing. The latter procedure involves field cooling in simultaneously applied parallel (circles) and antiparallel (squares) magnetic and electric fields of $B= \pm 0.05 \mathrm{~T}$ and $\mathrm{E}=1 \mathrm{~V} / 103 \mathrm{~nm}$. Cooling takes place from $400 \mathrm{~K}$ down to $20 \mathrm{~K}$. The subsequent measurements of the polar Kerr signal are performed on heating in $\mathrm{B}=\mathrm{E}=0$. The presence of a remanent Kerr signal is at first surprising and indicates a non compensated remanent surface magnetic moment in a system which has AF spin ordering in the bulk. Our preliminary studies of the energetics and thermodynamics of the ordering of the $\mathrm{Cr}$ atoms at the $\mathrm{Cr}_{2} \mathrm{O}_{3}$ (111) surface predict a very unusual stability of an uncompensated magnetic surface moment when the film is prepared in its antiferromagnetic single domain state. ${ }^{28}$ The roughness insensitivity of the uncompensated surface moment is understood as a consequence of a specific $\mathrm{Cr}$ termination. The latter stabilizes the structure by avoiding a charged surface. Its magnetization is carried by the $S=3 / 2$ spins of the $\mathrm{Cr}^{3+}$ ions. The temperature dependent Kerr magnetometry evidences in addition that the surface magnetization undergoes a sequence of magnetic phase transitions which are very likely associated with structural surface transitions in the $100-300 \mathrm{~K}$ temperature range. The anomalies in the Kerr signal at about $T=130 \mathrm{~K}$ and $220 \mathrm{~K}$ (dashed vertical lines) match with those found in the LEED pattern of a $\mathrm{Cr}_{2} \mathrm{O}_{3}$ (111) surface and have been interpreted there as structural transitions from a $1 \times 1$ into a $\sqrt{3} \times \sqrt{3}$ structure and back. ${ }^{29}$ Details of these transitions as well as the strength of the low temperature remanent Kerr signal depend on the magnetoelectric annealing procedure and, hence, on the AF domain structure of the $\mathrm{Cr}_{2} \mathrm{O}_{3}$ film. This dependence indicates an electrically controlled surface and interface magnetism of $\mathrm{Cr}_{2} \mathrm{O}_{3}$. Note that the Néel temperature of $\mathrm{Cr}_{2} \mathrm{O}_{3}$ known in bulk to be $T_{\mathrm{N}}=307 \mathrm{~K}$ can also be identified in the remanent Kerr data (dashed vertical line at about $T=320 \mathrm{~K}$ ) when the film has been exposed to a magnetoelectric annealing procedure (circles and triangles). Details of the electric controlled surface magnetism and its unusual 
robustness against roughness will be discussed elsewhere. ${ }^{28}$ Electric control of the exchange bias is realized by replacing the passive antiferromagnetic pinning layer with a magnetoelectric antiferromagnet namely $\mathrm{Cr}_{2} \mathrm{O}_{3}$. $\mathrm{These}$ fascinating properties make $\mathrm{Cr}_{2} \mathrm{O}_{3}$ a prototypical material for potential spintronic applications.

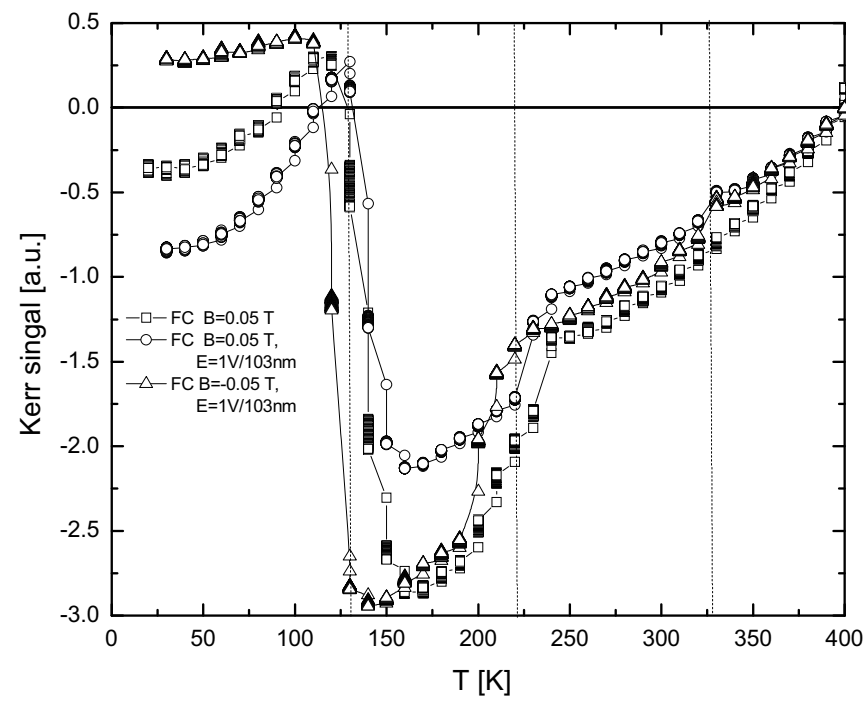

Fig. 2: (color online) Polar Kerr signal vs. temperature after field cooling the sample exclusively in an axial applied magnetic field of $0.05 \mathrm{~T}$ (squares) and after magnetoelectric annealing in simultaneously applied parallel (circles) and antiparallel (squares) magnetic and electric fields of $B= \pm 0.05 \mathrm{~T}$ and $E=9.7 \mathrm{MV} / \mathrm{m}$. Cooling takes place from $400 \mathrm{~K}$ down to $20 \mathrm{~K}$. The polar Kerr signal is measured on heating in $B=E=0$.

\section{Two-phase Multiferroic $\mathrm{BaTiO}_{3} / \mathrm{Fe}$ heterostructure}

Two-phase multiferroics combine ferroelectric and (anti)ferromagnetic thin films either in horizontal or vertical geometry. ${ }^{6,30}$ It is now well established that lattice distortions during the structural phase transitions of an underlying ferroelectric materials can cause changes in the resistivity and magnetic properties of a top magnetic layer. This was first demonstrated on a BTO/LSMO heterostructure ${ }^{31}$ and was recently confirmed in a variety of systems. ${ }^{16,32,33}$ The changes in magnetization and resistance are primarily attributed to the strain induced coupling at the interface. Until now, most studies are confined to heterosystems composed of compound FM materials and complex FE oxides. Recently we reported on the magnetic and magnetoelectric properties of a generic $\mathrm{BaTiO}_{3} / \mathrm{Fe}$ heterostructure where the individual material characteristics are well documented. ${ }^{34}$ Here we show new data correlating the structural and accompanying magnetic transitions with the electric resistance of the Fe film. In addition we deduce here from our experimental magnetization data the temperature dependence of the stress $\sigma$ induced by the BTO on the Fe layer. Moreover, we map the electric field dependence of the coercivity of $\mathrm{Fe}$ onto the polarization dependence of the coercivity via the polarization hysteresis of BTO.

Single crystal bulk $\mathrm{BaTiO}_{3}$ exhibits three distinct structural phase transitions: cubic-tetragonal (C-T) at a temperature $T \approx 393 \mathrm{~K}$, tetragonal-orthorhombic (T-O) at $T \approx 278 \mathrm{~K}$, and orthorhombic-rhombohedral (O-R) at $T \approx 190$ $\mathrm{K}$, respectively, which are well documented in literature. ${ }^{35,36}$ Similarly, Fe is a classical $3 \mathrm{~d}$ transition metal which has a Curie temperature $T_{\mathrm{c}}=1043 \mathrm{~K}$ and exhibits bcc structure in its most stable form.

We deposited $10 \mathrm{~nm}$ thick Fe film on a single-crystal $\mathrm{BaTiO}_{3}(100)$ substrate by molecular beam epitaxy at a base pressure of $5 \times 10^{-11}$ mbar. During growth of the Fe film, the substrate was heated to and maintained at $T=110^{\circ} \mathrm{C}$. This warrants the tetragonal structure of BTO and consequently maintains its ferroelectric polarity. $\theta-2 \theta$ X-ray diffraction and pole figure scans reveal the polycrystalline nature of the top Fe film. The magnetic and magnetoelectric properties of our BTO/Fe heterostructure were investigated using superconducting quantum interference device (SQUID) magnetometry (Quantum Design, MPMS XL) and magneto-optic Kerr effect (MOKE) technique. The resistivity 
properties were carried out by four-point technique in a closed cycle cryostat (Janis Research, CCS-350H). Note that during all measurements the magnetic filed is applied along the sample plane.

Figure 3 (a) shows the temperature dependence of magnetization, $M v s . T$, measured in a magnetic field of $\mu_{0} H$ $=2 \mathrm{mT}$ after cooling the $\mathrm{BTO} / \mathrm{Fe}$ heterostructure from $T=400 \mathrm{~K}$ to $T=5 \mathrm{~K}$ in a saturation field of $\mu_{0} H=0.8 \mathrm{~T}$. Here the in-plane component of the magnetization is displayed. Fig.3 (b) presents the temperature dependence of the resistance, $R$ $v s$. $T$, recorded in a magnetic field of $\mu_{0} H=200 \mathrm{mT}$. It is clearly noticed from the figure that $M v s . T$ and $R v s . T$ heating and cooling branches exhibit distinct anomalies and thermal hysteresis at structural transitions from $\mathrm{T}$ to $\mathrm{O}$ and $\mathrm{O}$ to $\mathrm{R}$ states of the BTO single crystalline substrate. This unequivocally indicates that the magnetic properties of the top Fe film are strongly modified owing to the in-plane lattice distortions of $\mathrm{BaTiO}_{3}$ during its structural phase transitions via magnetoelastic coupling at the interface. A quantitative correlation between $M v s$. $T$ and $R v s$. $T$ measurements is inconclusive at this point and needs further investigation. The linear $R$ vs. $T$ behavior before and after the $\mathrm{R}$ to $\mathrm{O}$

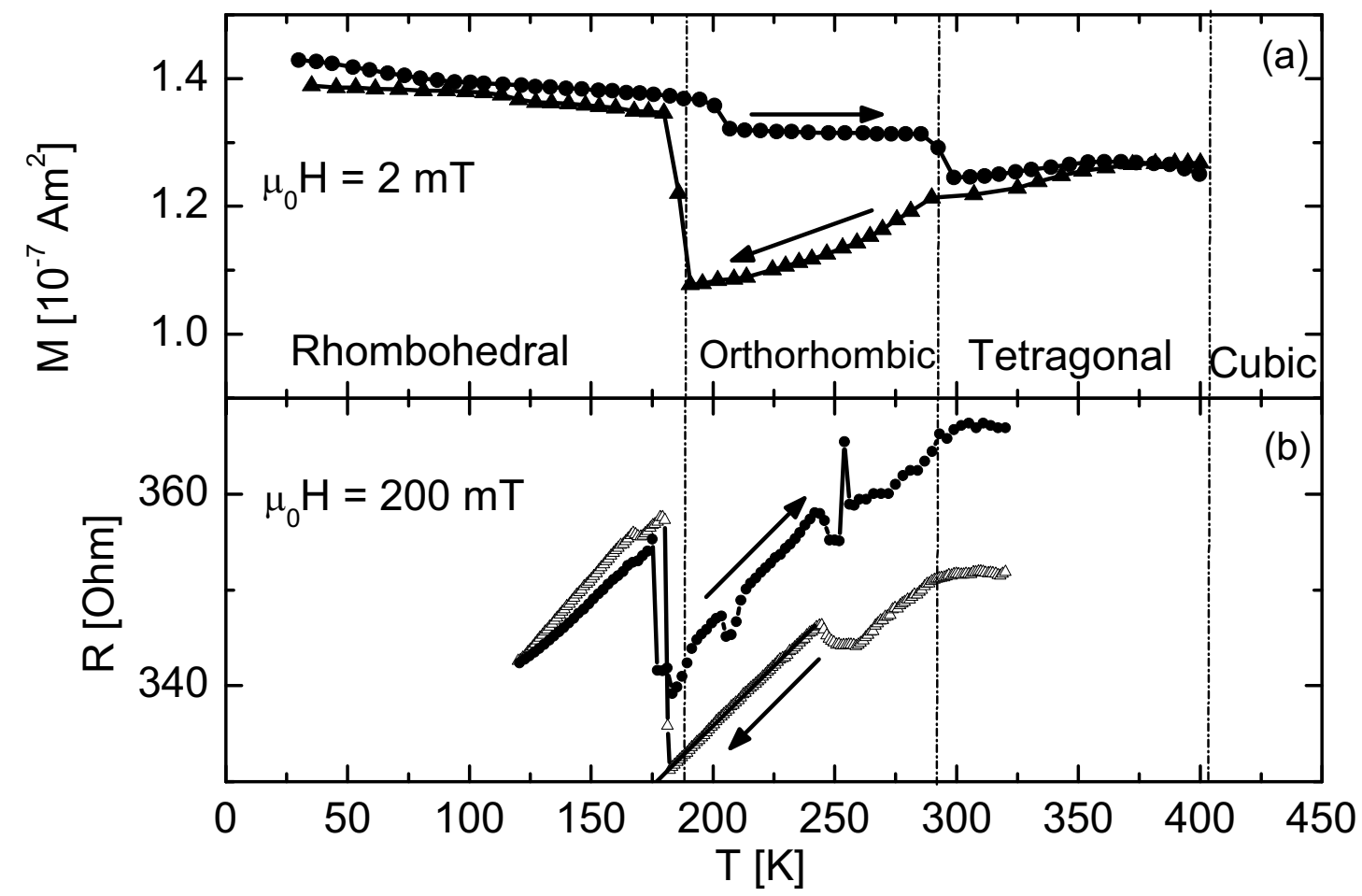

Fig. 3: (color online) (a) Temperature dependence of in-plane magnetization at a magnetic field of $\mu_{0} H=2 \mathrm{mT}$. Arrows point to the direction of temperature scans. Letters R, O, T, and C denote the Rhombohedral, Orthorhombic, Tetragonal, and Cubic states of $\mathrm{BaTiO}_{3}$ single-crystal substrate, respectively. Dotted lines distinguish respective transitions. (b) Resistance vs. temperature at $\mu_{0} H=200 \mathrm{mT}$. Arrows and dashed lines have the same meaning as in (a). Solid line is a linear fit.

transition hints at the typical metallic linear electron phonon scattering contribution to the resistance. A linear fit (solid line Fig 3 (b)) reveals $d \rho / d T \approx 10^{-9} \Omega m / K$ where $\rho$ is the specific resistance of our Fe film of lateral dimensions $4 \times 5$ $\mathrm{mm}^{2}$ and $10 \mathrm{~nm}$ thickness.

We now consider the $M$ vs. $T$ data to analyze the temperature dependent stress $\sigma$ vs. $T$ which the $\mathrm{BaTiO}_{3}$ substrate imposes on the Fe film. The analysis uses the Stoner-Wohlfarth relation between anisotropy and coercivity. We use our experimental coercivity data and correlate them with the stress dependent effective anisotropy allowing to deduce $\sigma$ vs. $T$. In order to derive the effective anisotropy we consider first the magnetoelastic energy which is given 
by $^{37} \mathrm{E}_{\mathrm{me}}=\mathrm{K}_{\mathrm{me}} \sin ^{2} \theta$, where the stress anisotropy constant $K_{\mathrm{me}}$ reads $\mathrm{K}_{\mathrm{me}}=\frac{3}{2} \lambda \sigma \cdot \lambda$ is the average magnetostriction coefficient quantifying the relative length change of the sample between the demagnetized and magnetized states and $\sigma$ the induced stress. $\theta$ is the angle between the magnetization $M$ and the direction of non vanishing $\sigma$ (see inset Fig. 4 (b)). Apparently $\mathrm{K}_{\mathrm{me}}>0$ favors $\theta=0$ which means parallel alignment of the magnetization relative to the stress axis while $\mathrm{K}_{\mathrm{me}}<0$ favors perpendicular alignment with $\theta=\pi / 2$.

The effective anisotropy constant, $K_{\text {eff }}$, of Fe in contact with BTO is the sum of several anisotropy contributions and can be expressed as

$$
\mathrm{K}_{\mathrm{eff}}=\mathrm{K}_{\mathrm{u}}+\frac{2 \mathrm{~K}_{\mathrm{s}}}{\mathrm{t}_{\mathrm{Fe}}}-\frac{\mu_{0}}{2} \mathrm{M}_{\mathrm{s}}^{2}+\mathrm{K}_{\mathrm{me}}
$$

where $K_{u}$ is the volume magnetocrystalline anisotropy constant, the second term is the surface anisotropy with its constant $K_{s}$ for a film thickness of $t_{\mathrm{Fe}}$, the third term is the shape anisotropy which favors an easy in-plane magnetization, and the last term is the magnetoelastic anisotropy term, $K_{m e}=\frac{3}{2} \lambda \sigma$.

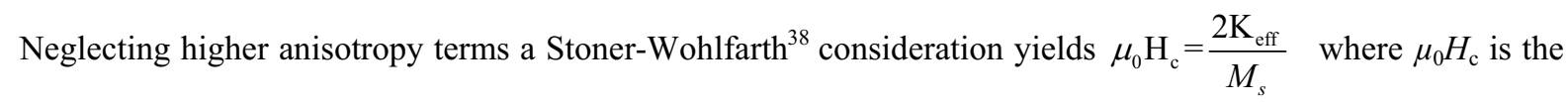
coercivity. Substituting the latter coercivity expression into Eq.(1) one obtains after some rearrangements

$$
\sigma(T)=\frac{\mu_{0} M_{s} H_{c}(T)-2\left(K_{u}+\frac{2 K_{s}}{t_{F e}}-\frac{\mu_{0}}{2} M_{s}^{2}\right)}{3 \lambda} .
$$

In-plane magnetic hysteresis loops, $M v s . \mu_{0} H$, were measured (see Fig. 4 (a) for selected loops) at different temperatures in the $\mathrm{T}, \mathrm{O}$, and $\mathrm{R}$ phases revealing profound changes in the coercivity, $\mu_{0} H_{\mathrm{c}}$ as the input for Eq.(2). Inserting $K_{\mathrm{u}}=4.8 \times 10^{4} \mathrm{Jm}^{-3}$ [Ref. 37] and $K_{\mathrm{s}}=2 \times 10^{-5} \mathrm{Jm}^{-2}$ [Ref. 39] into Eq.(2), we obtain $\sigma(T)$ as shown in Fig.3. Note that at saturation fields of $\mu_{0} H_{\mathrm{s}}>30 \mathrm{mT}$ the magnetostriction coefficient of polycrystalline $\mathrm{Fe}$ is $\lambda \approx-7 \times 10^{-6}$ [Ref. 37]. In order to achieve a more quantitative understanding of the $M$ vs. $T$ data shown in Fig. 3 (a), a change in the sign of the stress changing the characteristics of the stress from compressive to tensile has to be inferred. ${ }^{34}$ Our analysis displayed in Fig. 4(b) evidences this non trivial change in the sign of $\sigma$ around the O-R transition. 


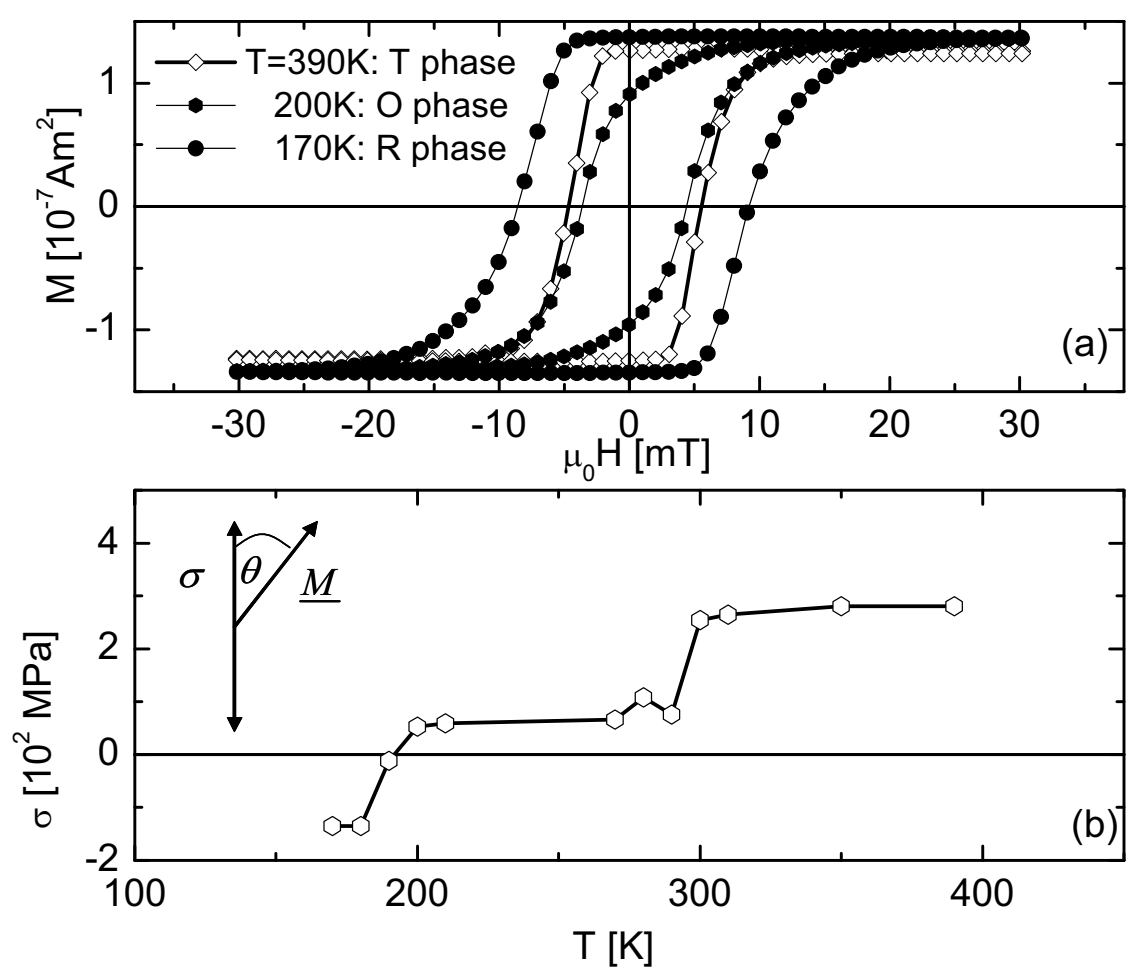

Fig. 4: (color online) (a) Magnetic hysteresis loops at selected temperatures $170 K \leq T \leq 390 K$. (b) Stress $\sigma$ vs. temperature deduced from Eq. (2) using the coercivity data of the hysteresis loops as experimental input. The inset in (b) shows the magnetization $M$ making an angle $\theta$ with the stress axis.

Spintronic devices based on the voltage control of magnetic properties will be extremely attractive for applications because of lower power consumption compared to their current-controlled counterparts. In Fig. 5(a) we show the longitudinal magneto-optic Kerr (L-MOKE) loops measured on our biferroic $\mathrm{BaTiO}_{3} / \mathrm{Fe}$ heterostructure. It clearly reveals the control of coercivity $\mu_{0} H_{\mathrm{c}}$ by the application of an electric field, $E$. The electric fields was changed from $E=-10 \mathrm{kV} / \mathrm{cm}$ up to $E=10 \mathrm{kV} / \mathrm{cm}$ and then descending back to $E=-10 \mathrm{kV} / \mathrm{cm}$. From Fig. $5(\mathrm{~b})$, it is seen that $\mu_{0} H_{\mathrm{c}}$ maximizes in the depolarized state of $\mathrm{BaTiO}_{3}$ and drops symmetrically by $\left|\Delta H_{\mathrm{c}} / H_{\mathrm{c}}\right| \approx 20 \%$ for $|E|$ above the ferroelectric coercivity of single crystal $\mathrm{BaTiO}_{3}$ substrate. ${ }^{40}$

We map the electric field dependence of the coercivity of Fe shown in Fig. 5 (b) onto the polarization dependence of the coercivity via the polarization hysteresis of BTO shown in Fig. 5 (c). This analysis shows in more detail that the major changes of the magnetic coercivity take place when polarization of BTO changes significantly which happens in the vicinity of the ferroelectric coercivity. There the coercivity change is significantly larger than those recently reported in FePt and FePd thin films immersed in an electrolyte. ${ }^{41}$ Apart from the fact that our FM electrode is simpler and the electrical control of the coercivity is much larger, an all solid state structure is by far more attractive for novel magnetostrictive memory device ${ }^{42}$ and multifunctional device applications. ${ }^{4,5,7,27,30}$

The change in $\mu_{0} H_{\mathrm{c}} v s . E$ originates from the magnetoelastic anisotropy due to out-of-plane lattice distortions in $\mathrm{BaTiO}_{3}$ crystal. As a consequence of this, the $E$-dependence of stress can be calculated from $\Delta \sigma(E)=\sigma(E)-\sigma(E=0)=\mu_{0} M_{s} H_{c} / 3 \lambda$. We find $\Delta \sigma(E=4 \mathrm{kV})=0.9 \times 10^{5} \mathrm{~Pa}$. Note that the stress induced by the electric field is much smaller than the stress induced by temperature change shown in Fig. 4(b). Finally we can estimate the maximum magnetoelectric susceptibility which reaches a giant value of $\alpha=\mu_{0}(\partial M / \partial E)_{\mid H=H_{c}}=1 \times 10^{-6} \mathrm{sm}^{-1}$ just at $H=H_{\mathrm{c}}{ }^{16}$

7036-32 V. 2 (p.7 of 9) / Color: No / Format: Letter / Date: 7/8/2008 3:57:50 PM 

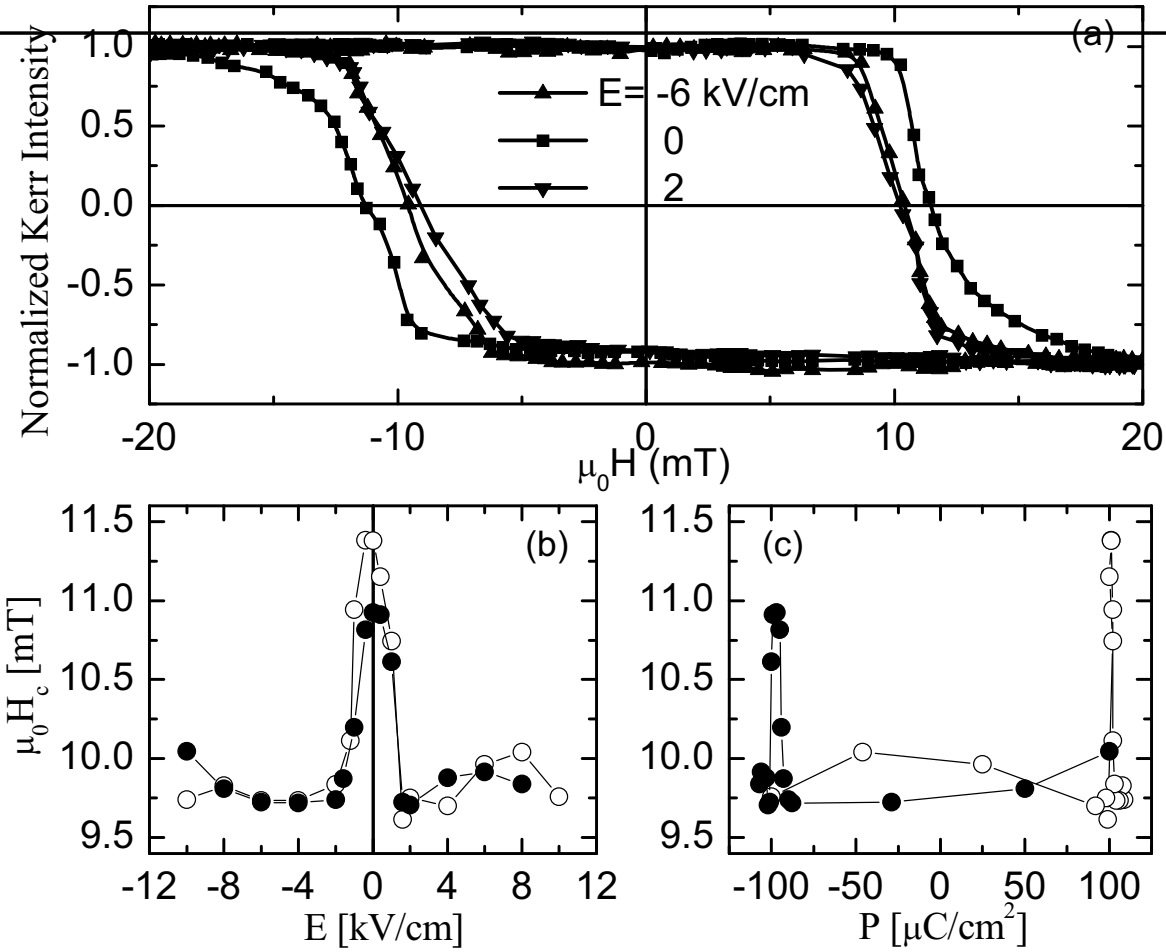

Fig. 5: (color online) Normalized Kerr magnetic loops at room temperature measured at different applied electric fields $-10 \mathrm{kV} / \mathrm{cm}<E<10 \mathrm{kV} / \mathrm{cm}$. (b) $\mu_{0} H$ vs. $E$ as obtained from loops in (a). Arrows point to directions along which the change of electric fields takes place in steps. (c) Polarization $v s$. electric field.

\section{Conclusions}

We reported recent results on the electrically controlled magnetism in the classical magnetoelectric antiferromagnet $\mathrm{Cr}_{2} \mathrm{O}_{3}$ thin film and a multiferroic systems $\mathrm{BaTiO}_{3} / \mathrm{Fe}$ heterostructure. Drastic deviations from the bulk characteristics are evidenced in $\mathrm{Cr}_{2} \mathrm{O}_{3}$ (111) textured thin films. Specific surface magnetic states and their electric field control make these films potential candidates for future spintronics applications. Further, we evidenced that the magnetic properties of a simple $3 \mathrm{~d}$ transition metal ferromagnetic thin film can be strongly altered in proximity with a single-crystal ferroelectric. We demonstrate electric and thermal control of magnetic anisotropy and coercivity by studying a classical ferroelectric-ferromagnetic $\mathrm{BaTiO}_{3} / \mathrm{Fe}$ heterostructure. The fingerprints in the electric resistance of the magnetic transitions data are reported. The stress imposed by the $\mathrm{BaTiO}_{3}$ substrate on the Fe film is deduced and the magnetoelectric susceptibility in the vicinity of the coercive field has been estimated and found to be giant. Simplicity of our all solid state heterostructure and strength of the observed effects promise significant potential for future spintronic applications.

This work is supported by NSF through Career DMR-0547887, MRSEC DMR-0213808, and Nebraska Research Initiative. 


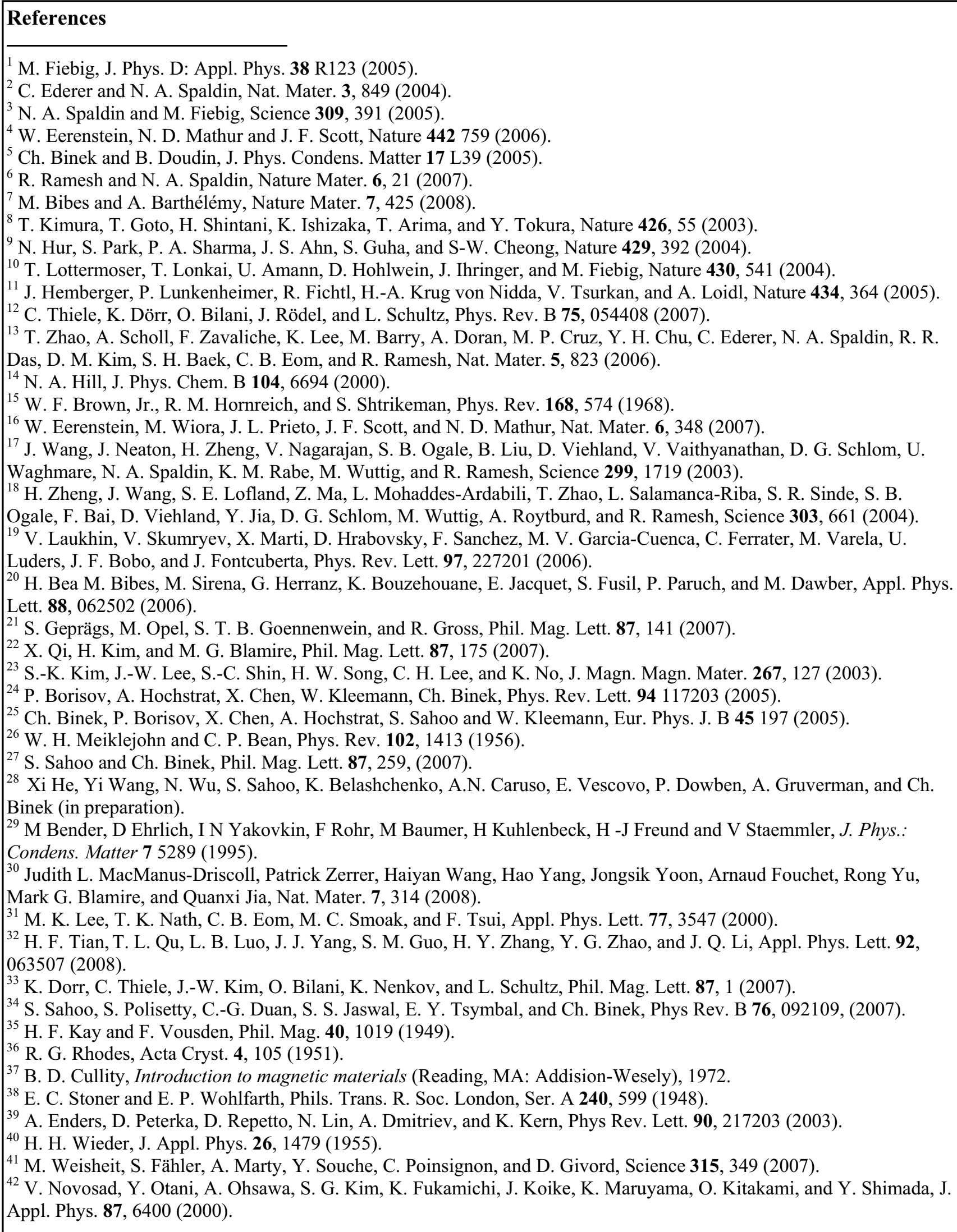

